\title{
Ist ein jährliches Screening bei Rauchern sinnvoll?
}

\author{
Das Screening von Rauchern mittels Low-Dose- \\ Computertomografie (LDCT) ist in seinem positiven Effekt auf \\ die Mortalität mit dem einer langjährigen Nikotinabstinenz \\ vergleichbar, wie eine retrospektive Analyse von Nichole T. \\ Tanner et al. nun gezeigt hat. \\ Am J Respir Crit Care Med 2016; 5: 534-541
}

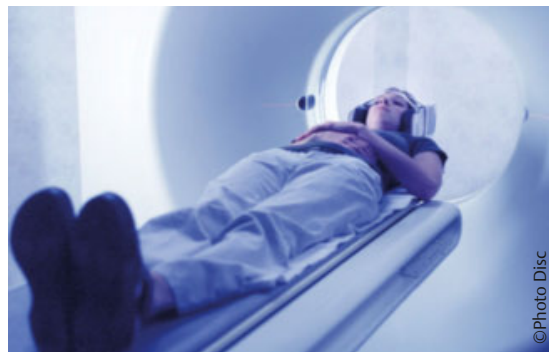

Trotz der massiven gesundheitlichen Probleme, die mit dem weltweiten Nikotinabusus assoziiert sind, fehlen immer noch valide epidemiologische Daten zur Effektivität präventiver Maßnahmen. Dies betrifft sowohl Interventionsprogramme (Abstinenz), als auch diagnostische Methoden zur frühen Detektion eines Lungentumors. Hier ist besonders das LDCT von Relevanz, da dieses Screeninginstrument zunehmend verfügbar wird. Unklar ist auch, welche Synergieeffekte durch die Kombination von Intervention und radiologischem Screening erzielt werden.

Zur Analyse standen den Autoren die Registerdaten des National Lung Screening Trials (NLST) zur Verfügung. Sie umfassen die klinischen Verläufe von 50263 Probanden. Davon hatten 26073 den Nikotinkonsum im Mittel seit 7 Jahren beendet. Bei allen anderen bestand weiterhin ein Abusus. Unabhängig vom Nikotinstatus fand bei einer Hälfte ein jährliches radiologisches Screening mittels LDCT statt. Bei allen anderen wurde unregelmäßig ein Röntgenthorax angefertigt. Um die statistische Adjustierung zu gewährleisten, wurden auch wichtige Komorbiditäten und sozio-ökonomische Daten mit berücksichtigt. Zielkriterium der Studie war die lungenkarzinomspezifische Mortalität in Abhängigkeit von der eingesetzten präventiven Strategie.
Ein gegenwärtiger Nikotinabusus verdoppelte das Risiko, an einem Lungenkarzinom zu versterben (HR 2,2). Ferner zeigte sich, dass 7 Jahre Nikotinabstinenz (mindestens 30 Packungsjahre) diese Mortalität um 20\% verminderte. Das LDCT-Screening bei Rauchern hatte einen ähnlich günstigen Effekt auf die Tumormortalität (15\%). Die Kombination (Abstinenz und LDCT) dagegen reduzierte das spezifische Mortalitätsrisiko um bis zu $38 \%$. Im Zeitverlauf erniedrigte die Nikotinabstinenz das Mortalitätsrisiko jährlich um $6 \%$. Ein zusätzliches radiologisches Screening verminderte dieses Risiko um weitere $3 \%$. Der zusätzliche, präventive Effekt des LDCT-Screenings war auch noch 15 Jahre nach Abstinenzbeginn nachweisbar.

Die Autoren verweisen in diesem Zusammenhang allerdings auf die hohen Kosten, die sich durch das CT-Screening ergeben. So verursacht ein durch Interventionsprogramme gewonnenes Lebensjahr in guter Qualität (QALY) durchschnittliche Kosten von etwa 1000 bis 4500 US\$. Das durch LDCT-Screening gewonnene QALY dagegen muss mit 81000 US\$ angesetzt werden.

\section{Fazit}

Bei Rauchern sollte ein regelmäßiges radiologisches Screening mittels LDCT erwogen werden. Dessen positiver Effekt auf die Mortalität entspricht dem einer jahrelangen Abstinenz. Optimal ist nach Angaben der Autoren allerdings die Kombination von Abstinenz und anschließenden, langjährigen LDCT-Kontrollen. Hierdurch würde sich bei Exrauchern der Mortalitätsgewinn verdoppeln.

\section{Dr. Horst Gross, Berlin}

